\title{
Recent Advances in Androgen-Receptor Splicing Variants Related to the Mechanism and Treatment of Castration-Resistant Prostate Cancer
}

\author{
Jianguo Liao ${ }^{1}$, Wenhai $\operatorname{Pan}^{1}$
}

\author{
${ }^{1}$ Department of Urology, Guangzhou Red Cross Hospital, Jinan University, Guangzhou, China
}

\begin{abstract}
Introduction
Prostate cancer is one of the most common malignant solid tumors in men. In the United States in 2020, prostate cancer is predicted to cause 191930 new cases, accounting for $21 \%$ of the total number of new solid tumors in all men. [1] China and Asia faster-growing incidence of prostate cancer ( $\mathrm{PCa}),[2]$ The death rate from prostate cancer in urban areas of mainland China increased by nearly 50\% in 2009, compared with 2004 . At present, there are significant regional differences in the detection of prostate cancer in China, and the patients with advanced metastatic prostate cancer account for a large proportion in the whole patient population. [3]-[6] Although endocrine therapy is the standard regimen for the treatment of advanced metastatic prostate cancer, almost all patients will eventually develop drug resistance and enter Castration-resistant prostate cancer (CRPC) after receiving endocrine therapy within 18 to 36 months, [7] resulting in significantly increased mortality and poor quality of life. Therefore, more in-depth research is needed in the mechanism and treatment of CRPC. Androgen receptor(AR) is the focus of research, and androgen receptor splicing variant(AR-Vs) is a new and important breakthrough point. This review provides a theoretical basis for further research on androgen receptor splicing variants related to the mechanism and treatment of CRPC.
\end{abstract}

\subsection{Diagnostic Criteria of CRPC}

In the definition of CRPC, "castration" includes surgical castration and drug castration. Regardless of the castration mode, $95 \%$ of the androgens will be removed in the future from the hypothalamic-pituitary-gonadal axis. Diagnosis of CRPC in the 2017 edition of the European Association of Urology (EAU) guidelines includes three criteria: (1) Serum testosterone reaching castration level $(<50$ ng/dI, or < $1.7 \mathrm{nmol} / \mathrm{L}) ; \quad$ (2)Prostate-specific antigen(PSA) progress: 3 times in a row during the interval of 1 week, the PSA increased by more than $50 \%$ from the minimum value, and the absolute value of PSA increase was $>2 \mathrm{ng} / \mathrm{mI}$. ; (3)Imaging progress: bone scan found two or more bone metastatic lesions or large soft tissue lesions meeting Response Evaluation Criteria in Solid Tumors . [8] The CRPC consists of the following 2 stages: (1)Androgen independent and AR dependent stages; (2)Androgen independent and AR independent stages.[9]

\begin{abstract}
2..2 Androgen Receptor (AR) and Androgen Receptor splicing Variant (AR-Vs)

Androgen receptor (AR) is a member of the steroid hormone receptor family. [11] It contains n-terminal trans-activation domain (encoded by exon 1), DNA binding domain (encoded by exon 2 and 3), short hinge region (encoded by exon 4), and c-terminal ligand-binding domain (LBD; Exon 4-8 encoding), [12]AR is activated by binding to androgen, and plays a key role in male physiology and pathology, involved in all stages of prostatic tumor initiation, development, and therapeutic resistance. [10] Prostate cancer by a variety of mechanisms such as AR gene amplification, mutation and steroid metabolism, cell signal transduction and regulation of the change of the core protein produces resistance to androgen deprivation therapy (ADT). [13] The expression of androgen receptor splicing variants (AR - VS)lacking ligand-binding domain is a kind of new mechanism.[7] The discovery of the constitutive active AR splicing variant has become a new clinical trial and novel method for targeting AR.[13]
\end{abstract}

As a truncated subtype of AR, the androgen-receptor splicer variant (AR-Vs) lacks ligand-binding domain and still has constitutive activity in the absence of circulating androgens. [14] It activates AR reporter genes in the absence of ligand, thereby promoting the progression of prostate cancer. [17][18] Compared with primary prostate cancer, the expression of androgen-receptor splicing variant with truncated C terminal in CRPC was significantly increased. [15] the expression of AR-Vs AR-V1, AR-V7 and AR-V567es) in CRPC bone metastasis was increased and indicated poor prognosis. [19] Ar-v7 in androgen receptor splicing variant was particularly important, and more and more evidences emphasized that the expression of AR splicer variant could be used as a potential 
prediction biomarker and a therapeutic target for advanced prostate cancer. [16] [17]

\section{Several androgen-receptor splicer variants that play an important role in CRRP \\ 3.1 Androgen receptor splicing variant \\ 567es( ARv567es)}

ARv567es is produced by splicing the 5th, 6th and 7th exons of AR. [22] The expression of ARv567es can often be detected in human metastatic prostate cancer. [19][20]ARv567es can induce castration resistance in prostate tumors of human xenograft, and the ratio of androgen receptor full length(ARfl) expression is positively correlated with castration resistance. [22] In the standard taxane treatment of castration-resistant prostate cancer (CRPC), the effect of docetaxel treatment was significantly enhanced because ARv567, after interacting with the microtubules of prostate cells, increased the sensitivity of taxane-induced microtubules stabilization. [21]ARv567es can be used as a biomarker for early recurrence of CRRP and is associated with a particularly poor prognosis of CRRP. [19][22]ARv567es promotes the development of castration-resistant prostate cancer by inducing tumorigenesis de novo, [20] acting as constitutively active receptors, increasing the expression of androgen receptor full length(ARfl) and enhancing the transcriptional activity of AR. [22] [20]

\subsection{Androgen receptor Splicing Variant 7(AR-V7)}

Ar-v7 /AR3 is one of the major splicing variants expressed in castration-resistant prostate cancer tissues in humans. It has constitutive activity and its transcriptional activity is not regulated by androgens or antiandrogens. [23] Under the condition of androgen deprivation and the absence of ligand, AR-V7 in the nucleus of $\mathrm{PCa}$ can drive the expression of classical androgen reactive gene and endue castration resistance to prostate cancer. [27] [18] [23]

During the progression of $\mathrm{PCa}$, especially in hormone-refractory prostate cancer (HRPC), AR-V7 was significantly up-regulated and predicted biochemical recurrence after surgical treatment $(\mathrm{P}=$ 0.012). [31][23][27] The increased expression of AR-Vs (AR-V1, AR-V7 and AR-V567es) in bone metastasis in CRPC not only indicates the correlation with advanced bone metastasis of prostate cancer, but also indicates a very poorprognosis.[19]

\subsubsection{AR-V7 is related to endocrine therapy for castration-resistant prostate cancer}

Compared with hormone-sensitive prostate cancer, AR-V1 and AR-V7 in HRPC were significantly increased in both protein expression and mRNA expression, the mRNA expression of more than 20 times $(n=25),[24]$ [27] especially after treatment with abiraterone or enthraludine, the expression of ar-v7 was more significant, ar-v7 was associated with decreased response to castration-resistant prostate cancer (CRPC) endocrine therapy and decreased overall survival.[31]
In 168 cases treated with androgen deprivation (ADT) prostate cancer patients showed that the positive expression of AR-V7 significantly reduced the response rate of prostate-specific antigen (PSA) ( $\mathrm{P}<$ 0.001 )to androgen deprivation therapy and significantly reduced progression-free survival $(\mathrm{P}<0.0001)$ and overall survival of castration-resistant prostate cancer(HR: 4.826; 95\% CI: 2.960-7.869; P < 0.001). [7]

Among the patients with metastatic castration resistant prostate cancer treated with enzalurudine or abirutrone, the PSA response rate, prostate specific antigen progression-free survival(PSA PFS) and overall survival (OS) of AR-V7-positive patients were significantly lower than those of AR-V7-negative control group.[31] [30] [34] In the enzalutamide group,AR-V7-positive patients had a PSA response rate $(0 \%$ vs. $53 \%, \mathrm{P}=0.004)$, PSA PFS (median 1.4 months vs. 6.0 months, $\mathrm{P}<0.001)$, no clinical or radiological progression (median 2.1 months vs. 6.1 months, $\mathrm{P}$ $<0.001$ ), and OS (median 5.5 months vs. unmet, $\mathrm{P}=$ 0.002)), relative to AR-V7-negative patients. Also in treatment of male abiraterone, AR-V7--positive patients had a PSA response rate $(0 \%$ vs. $68 \%, \mathrm{P}=$ $0.004)$ ), PSA PFS (median 1.3 months vs. unmet, $\mathrm{P}=$ 0.001 ), no clinical or radiological progression (median 2.3 months vs. unmet,P <0.001), and OS (median 10.6 months vs. unmet,P $=0.006)$ ), relative to AR-V7--negative patients. In patients with metastatic castration-resistant prostate cancer treated with enzalutamide or abiraterone, AR-V7- is not only an independent predictor of PSA response rate and PFS, [30] but is also associated with resistance to enzalutamide or abiraterone in castration-resistant prostate cancer. [30] [28] [34]

\subsubsection{AR-V7 is related to taxane chemotherapy in castration-resistant prostate cancer}

Although AR-V7 expression of Circulating Tumor Cells (CTC) in metastatic castration-resistant prostate cancer(mCRPC) was not associated with major resistance to taxane chemotherapy, [35] it was shown in 37 metastatic CRPC patients treated with taxane (docetaxel or cabataxel) and 62 CRRP patients treated with enzalutamide or abirirone: In the AR-V7 negative patients, there was no difference in the outcomes of the three treatments, while in the AR-V7positive patients, the PSA response rate,PSA PFS and PFS of the taxanes treatment group were all higher than that of the enzalurudine treatment group or the abiraterone treatment group,with Prostate-specific antigen responses (41\% vs $0 \%, \mathrm{P}<.001)$, PSA PFS hazard ratio [HR] 0.19 [95\%ci, 0.07-0.52] P = 0.001,PFS HR 0.21 $[95 \%$ ci, $0.07-0.59], \quad \mathrm{P}=0.003), \quad[35]$ when positiveAR-V7 was detected in circulating tumor cells (CTCS) before treatment, the risk of death of choosing taxanes was lower than that of using androgen receptor signaling (ARS) inhibitors (HR, 0.24; 95\% CI, 0.10-0.57; $\mathrm{P}=0.035)$.[29] In summary, the clinical outcome of taxanes was better than that of 
enzalutamide or abirirone, and AR-V7detection could be used as a treatment biomarker in CRPC. [35] AR-v7 is also a dynamic marker in abiritron, enzalutamide endocrine therapy, or taxane chemotherapy. "AR-V7can be transformed into a positive or negative state", and the continuous AR-V7 test in blood provides a method to observe the evolution of tumors in real time. [33]

\subsubsection{Mechanism of AR-V7 induced castration resistance in CRRP}

Under the condition of ADT enhancement, increased AR the interaction between the pre - mRNA and splicing factor, increase RNA splicing enhancer and its binding protein (U2AF65 and ASF/SF2) to AR - V7 3 'splice sites raise, lead to the increase of AR - V7 level in prostate cancer cells .[24]Although the expression of AR - V7 protein relative to AR - FL is low, the low level of AR - V7 are not sufficient to restore the AR activity, but after androgen deprivation induced by fast, [26] the increased ar-v7 was mediated by AR-fl to endue ligand-independent trans-activation of AR, [32] enabling the tumor to retain the basic AR activity needed for survival until a more effective mechanism for AR activation was arise.[26] AR3 may directly raise AKT1 expression, [23] regulate a variety of tumor autocrine/paracrine factor (Tgf beta 2 and Igf1), raised some epithelium - interstitial conversion related genes such as single or superposition approach to driving of castration resistant prostate cancer. [25] In CRRP, the expression of ar-v7 is positively correlated with UBE2C. [14]

\subsection{AR4,AR5 and AR45}

AR4 and AR5 lacking ligand-binding domains are two novel AR splection variants in hormone-insensitive prostate cancer cells. [23]AR45 is a natural variant of the human androgen receptor (AR). AR45 is mainly expressed in the heart and skeletal muscle. AR45 can inhibit the function of $\mathrm{AR}$ and reduce the proliferation rate of androgen-dependent LNCap cells. [36]

\subsection{ARV1, ARV9, ARV12, ARV13 and ARV14}

ARV1 and ARV9 are similar in structure, both of which do not possess the basic amino acids of the two-part nuclear localization sequence, and can be conditionally activated according to the level of androgens. ARV13 and ARV14 had no functional effect, and ARV12 had constitutive activity. The expression levels of ARV12 and ARV9 in CRPC specimens were significantly increased, and ARV12 was positively correlated with patients with Gleason score of 8 or above $(\mathrm{P}=0.017)$. [37]

\subsection{AR8}

AR8 is a new AR splicing variant, mainly located on the plasma membrane, upregulation in castration-resistant prostate cancer cells. AR8 promotes the binding of Src and AR to the EGF receptor in response to EGF treatment and enhances the tyrosine phosphorylation of AR to promote the progression of prostate cancer in the context of androgen deprivation.
[38]

\section{Conclusion}

At present, significant breakthroughs have been made in the research on the mechanism and treatment of prostate cancer, especially in the aspects of endocrinology and chemotherapy, which have improved the overall survival time and quality of life for patients. However, when prostate cancer inevitably progresses to the castration resistance stage, the prognosis is still extremely poor. [7]In CRRP, AR-Vs (AR-V7,AR-V567es) was significantly correlated with overall survival, progression-free survival, postoperative biochemical recurrence, and advanced bone metastasis. This paper preliminarily demonstrated the mechanism of AR-Vs in the treatment of abiraterone and enzalutamide resistance in castration-resistant prostate cancer, and also provided directional selection for the treatment of abiraterone, enzalutamide and taxo. [29][35]However, the specific mechanism of ar-vs in CRRP still needs to be further studied. It is expected that ar-vs can be used as a therapeutic target to develop effective target inhibitors with little side effects, so as to improve the outcome of poor prognosis of CRRP and improve the overall survival and quality of life of patients. AR-Vs is the key research direction in the future.[16][17]

The authors declare no conflict of interest.

\section{References}

1. Siegel RL, Miller KD, Jemal A Cancer statistics, 2020.CA Cancer J Clin. 2020 Jan;70(1):7-30. doi: 10.3322/caac.21590. Epub 2020 Jan 8.

2. YANG Jinyi ,YANG Mingzhoum WEI Weim ,YUE Wenchang . Epidemiological study on the occurrence and development of prostate cancer .Journal of Clinical Urology ,DOI 10.13201/j.issn.1001-1420.2017.09.018

3. Ye Dingwei, Zhu Yao. Epidemiology of prostate cancer in China: an overview and clinical implication. Chinese Journal of Surgery, DOI 10.3760/cma.j.issn.0529-5815.2015.04.003

4. HAN Sujun, ZHANG Siwei, et al. Analysis of the status and trends of prostate cancer incidence in China .Chinese Clinical Oncology,DOI 10.3969/j.issn.1009-0460.2013.04.009

5. Chinese Anticancer Association Genitourinary Oncology Committee .Chinese experts consensus on the treatment of metastatic prostate cancer 2018 edition, Chinese Journal of Surgery, DOI 10.3760/cma.j.issn.0529-5815.2018.09.002

6. ZENG Hao , CHONG Tie,et al. Assessment of updated guidelines for castration-resistant prostate cancer-Western China expert consensus conference, Journal of Modern Urology, DOI10.3969/j.issn.1009-8291.2017.02.002

7. Li H, Wang Z, Xiao W, Yan L, Guan W, Hu Z, et al. Androgenreceptor splice variant-7-positive prostate cancer: a novel molecular subtype with markedly worse androgen-deprivation therapy outcomes in newly diagnosed patients. Mod Pathol 2018;31:198-208.

8. CornfordP, BellmuntJ, BollaM, BriersE.EAU-ESTRO-SIOG G uidelines on Prostate Cancer. Part II: Treatment of Relapsing, Metastatic, and Castration-Resistant Prostate Cancer.Eur Urol. 2017 Apr;71(4):630-642

9. RASSO CS, wu YM, ROBINSON DR, et a1. The mutational landscape of lethal castration-resistant prostate cancer. Nature, 2012, 487(7406): 239-243.

10. Centenera MM, Harris JM, Tilley WD, Butler LM.The contribution of different androgen receptor domainsto receptor dimerization and signaling.Mol Endocrinol. 2008 Nov;22(11):2373-82

11. Narayanan R, Coss CC, Dalton JT.Development of selective androgen receptor modulators (SARMs). Mol Cell 
Endocrinol. 2018 Apr 15;465:134-142.

12. Philip A. Watson,Yinan F. Chen,Minna D. Balbas,John Wongvipat,Constitutively active androgen receptor splice variants expressed in castration-resistant prostate cancer require full-length androgen receptorProc Natl Acad Sci U S A. 2010 Sep 28; 107(39): 16759-16765.

13. Shafi AA, Yen AE, Weigel NL.Androgen receptors in hormone-dependent and castration-resistant prostate cancer.Pharmacol Ther. 2013 Dec;140(3):223-38.

14. Rong Hu, Changxue Lu, Elahe A. Mostaghel, Distinct transcriptional programs mediated by the ligand-dependent full-length androgen receptor and its splice variants in castration-resistant prostate cancer . Cancer Res. 2012 Jul 15; 72(14): 3457-3462.

15. Zhang X, Morrissey C, Sun S, Ketchandji M, et al.

16. Androgen receptor variants occur frequently in castration resistant prostate cancer metastases.PLoS One. 2011;6(11):e27970.

17. Ciccarese C, Santoni M, Brunelli M, et al.

18. AR-V7 and prostate cancer: The watershed for treatment selection?Cancer Treat Rev. 2016 Feb;43:27-35.

19. Paschalis A, Sharp A, Welti JC, Neeb A, et al.Alternative splicing in prostate cancer.Nat Rev Clin Oncol.

20. 2018 Nov;15(11):663-675.

21. Watson PA, Chen YF, Balbas MD, Wongvipat J, et al.Constitutively active androgen

22. receptor splice variants expressed in castration-resistant prostat e cancer require full-length androgen receptor.Proc Natl Acad Sci U S A. 2010 Sep 28;107(39):16759-65.

23. HörnbergE , Ylitalo EB, Crnalic S, et al. Expression of androgen receptor splice variants in prostate cancer bone metastases is associated with castration-resistance and short survival. PLoS ONE 2011;6:e19059.

24. Liu G, Sprenger C, Sun S, Epilepsia KS, Haugk K, et al.AR variant ARv567es induces carcinogenesis in a novel transgenic mouse model of prostatecancer.Neoplasia. 2 013 Sep;15(9):1009-17.

25. chongfuThadani-Mulero M, Portella L, Sun S, Sung M, Matov A, Vessella RL, et al. Androgen receptor splice variants determine taxane sensitivity in prostate cancer. Cancer Res 2014:74:2270-82.

26. Sun S, Sprenger CC, Vessella RL, et al. Castration resistance in human prostate cancer is conferred by a frequently occurring androgen receptor splice variant. J Clin Invest 2010;120:271530

27. Guo Z, Yang X, Sun F, Jiang R, Linn DE,.A novel androgen receptor splice variant is up-regulated during prostate cancer progression and promotes androgen depletion-resistant growth.Cancer Res. 2009 Mar 15;69(6):2305-2313.

28. Liu LL, Xie N, Sun S, Plymate S, et al.Mechanisms of the androgen receptor splicing in prostate cancer cells.Oncogene. 2014 Jun 12;33(24):3140-50.

29. Sun F, Chen HG, Li W, Yang X, Wang X, Jiang R..Androgen receptor splice variant AR3 promotes prostate cancer via modulating expression of autocrine/paracrine factors.J Biol Chem. 2014 Jan 17;289(3):1529-39.
30. Ziyang Yu, Sen Chen, Adam G. Sowalsky, et al.Rapid Induction of Androgen Receptor Splice Variants by Androgen Deprivation in Prostate CancerClin Cancer Res. 2014 Mar 15; 20(6): 1590-1600.

31. Hu R, Dunn TA, Wei S, et al. Ligand-independent androgen receptor variants derived from splicing of cryptic exons signify hormone-refractory prostate cancer. Cancer Res 2009;69:16-22.

32. Yingming $\mathrm{Li}$, Siu Chiu Chan,et al.Androgen receptor splice variants mediate enzalutamide resistance in castration-resistan prostate cancer cell lines Cancer Res. 2013 Jan 15; 73(2): 483-489.

33. Scher HI, Lu D, Schreiber NA, et al. Association of AR-V7 on Circulating Tumor Cells as a Treatment-Specific Biomarker With Outcomes and Survival in Castration-Resistant Prostate Cancer.JAMA Oncol. 2016 Nov 1;2(11):1441-1449

34. Seitz AK, Thoene S, Bietenbeck A, et al.

35. AR-V7 in Peripheral Whole Blood of Patients with Castration-r esistant Prostate

Cancer: Association with Treatment-specific Outcome Under A biraterone and Enzalutamide.Eur Urol. 2017 Nov;72(5):828-834

36. Sharp A, Coleman I, Yuan W, et al.Androgen receptor splice variant-7 expression emerges with castration resi stance in prostate cancer.J Clin Invest. 2019 Jan 2;129(1):192-208.

37. Mostaghel EA, Marck BT, Plymate SR, et al.

38. Resistance to CYP17A1 inhibition with abiraterone in castratio n-resistant prostate cancer: induction of steroidogenesis and and rogen receptor splice variants.Clin Cancer Res. 2011 Sep 15;17(18):5913-25

39. Nakazawa M, Lu C, Chen Y, Paller CJ, Carducci MA, Eisenberger MA, et al. Serial blood-based analysis of AR-V7 in men with advanced prostate cancer. Ann Oncol 2015;26:1859-65.

40. Antonarakis ES, Lu C, Wang H, Luber B, Nakazawa M, Roeser $\mathrm{JC}$, et al. AR-V7 and resistance to enzalutamide and abiraterone in prostate cancer. N Engl J Med 2014;371:1028-3

41. Antonarakis ES, Lu C, Luber B, Wang H, Chen Y, Nakazawa M, et al. Androgen receptor splice variant 7 and efficacy of taxane chemotherapy in patients with metastatic castrationresistant prostate cancer. JAMA Oncol 2015;1:582-91.

42. Ahrens-Fath I1, Politz O, Geserick C, Haendler B. Androgen receptor function is modulated by the tissue-specific AR45 variant.FEBS J. 2005 Jan;272(1):74-84.

43. Rong Hu, William B. Isaacs, and Jun Luo1,A Snapshot of the Expression Signature of Androgen Receptor Splicing Variants and Their Distinctive Transcriptional Activities Prostate. 2011 Nov; 71(15): 1656-1667.

44. Yang X, Guo Z, Sun F, et al. Novel membrane-associated androgen receptor splice variant potentiates proliferative and survival responses in prostate cancer cells. J Biol Chem 2011;286:36152-60

45. Dehm SM, Tindall DJ. Alternatively spliced androgen receptor variants. Endocr Relat Cancer 2011;18:R183-96. 